\title{
The detection of delirium in admitted oncology patients: a scoping review
}

\author{
Megan B. Sands ${ }^{1}(1) \cdot \operatorname{lan} W^{2} e^{2} \cdot$ Meera Agar ${ }^{3} \cdot$ Janette L. Vardy $y^{4,5}$
}

Received: 28 August 2021 / Accepted: 3 November 2021 / Published online: 15 January 2022

(c) Crown 2022

\section{Key summary points}

Aim To understand the validation of delirium detection tools in medical oncology, as well as identify data on incidence, prevalence and reversibility in this setting.

Findings Of twelve studies, only four used case ascertainment methods following published recommendations, six studies had a low risk of bias.

Message In delirium tool validation studies in the oncology setting, choice of appropriate gold standard for case ascertainment is a critical factor. New tools and new validations are not recommended, rather the critical application of existing tools depending on appropriate validation and clinical practicality for the setting.

\begin{abstract}
Purpose Delirium leads to poor outcomes for patients and careers and has negative impacts on staff and service provision. Cancer rates in elderly populations are increasing and frequently, cancer diagnoses are a co-morbidity in the context of frailty. Data relating to the epidemiology of delirium in hospitalised cancer patients are limited. With the overarching purpose of improving delirium detection and reducing the morbidity and mortality of delirium in cancer patients, we reviewed the epidemiological data and approach to delirium detection in hospitalised, adult oncology patients.

Methods MEDLINE, EMBASE, CINAHL, PsycINFO, and SCOPUS databases were searched from January 1996 to August 2017. Key concepts were delirium, cancer, inpatient oncology and delirium screening/detection.

Results Of 896 unique studies identified; 91 met full-text review criteria. Of 12 eligible studies, four applied recommended case ascertainment methods to all patients, three used delirium screening tools alone or with case ascertainment tools sub-optimally applied, four used tools not recommended for delirium screening or case ascertainment, one used the Confusion Assessment Method with insufficient information to determine if it met case ascertainment status. Two studies presented delirium incidence rates: 7.8\%, and $17 \%$ respectively. Prevalence rates ranged from $18-33 \%$ for general medical or oncology wards; $42-58 \%$ for Acute Palliative Care Units (APCU); and for older cancer patients: 22\% and 57\%. Three studies reported reversibility; $26 \%$ and $49 \%$ respectively (APCUs) and 30\% (older patients with cancer). Six studies had a low risk of bias according to QUADAS-2 criteria; all studies in the APCU setting were rated at higher risk of bias. Tool selection, study flow and recruitment bias reduced study quality.

Conclusion The knowledge base for improved interventions and clinical care for adults with cancer and delirium is limited by the low number of studies. A clear distinction between screening tools and diagnostic tools is required to provide an improved understanding of the rates of delirium and its reversibility in this population.
\end{abstract}

Keywords Delirium $\cdot$ Oncology $\cdot$ Cancer $\cdot$ Inpatient $\cdot$ Detection $\cdot$ Screening

1 University of New South Wales Prince of Wales Clinical School, Sydney, Australia

Presented in part at: Clinical Oncology Society of Australia Annual Scientific Meeting November 2019, European Association of Palliative Care Berlin May 2019, The American Delirium Society, San Francisco, CA June 11th 2018, Australian and New Zealand Society of Palliative Medicine Manly, NSW Australia September 6th 2018.

Megan B. Sands

meg.sands@unsw.edu.au
2 Singapore University Medical School, Singapore, Singapore, Singapore

3 University of Technology Sydney, Sydney, NSW, Australia

4 Concord Cancer Centre, Concord Repatriation General Hospital, Sydney, NSW, Australia

5 Sydney Medical School, University of Sydney, Sydney, NSW, Australia 


\section{Background and aim}

Delirium is a neurocognitive syndrome characterised by an altered level of arousal, altered awareness and cognition, and a reduced ability to direct, focus, sustain, and shift attention $[1,2]$. Delirium is associated with increased morbidity and mortality, longer length of stay [3, 4], and marked distress for cancer patients, their families and staff [5, 6]. Delirium is common in hospitalised patients [1,2], and outcomes can be improved via prevention $[7,8]$ and effective management [9]. The use of validated assessments improves detection and provides earlier identification of patients with delirium [10].

Under-diagnosis of delirium is an important issue in clinical settings [11]; outcomes are worse if the diagnosis of delirium is delayed or missed entirely [12]. Studies of general hospital patients indicate that pain, younger age, correct orientation in person, place and time, and previous psychiatric diagnosis, especially bipolar disorder or psychosis, are important risk factors for the diagnosis of delirium being missed [13]. One study has shown increasing age, poor performance on cognitive testing and lower serum albumin to be associated with a higher risk of delirium in the hospitalised cancer patients, however, less is known about factors which increases the misdiagnosis of delirium in cancer populations or whether there are specific clinical factors which can be used to mitigate risk [3].

The majority of epidemiological studies in delirium have targeted people over 65 years of age [14]. Although guidelines for the management of delirium in cancer settings exist [15], fewer studies have primarily focussed on adults (defined as 18yrs or older) in an acute hospital, oncology, inpatient setting [16-18]. More commonly studies including cancer patients have been in "stand-alone" palliative care units $[19,20]$, or subsets of cancer inpatient cohorts on the basis of palliative care [21-23] or psychiatry consultation/liaison services in acute hospitals [11]. A recent review of delirium in the palliative care setting yielded a point prevalence estimate of 35\% [95\% confidence interval $(C I)=0.29-0.40]$ at inpatient admission. [20] Studies indicate that in the palliative care cancer setting at least, whilst the prevalence of delirium is high, it remains reversible in approximately half of cases [24]. These data also lend support to the case for improved detection. Of interest, reversibility in the palliative care setting although not a universal possibility, has been associated with factors such as delirium aetiology specifically opioid, or other psychoactive medication, or dehydration, and where there is a less severe cognitive disturbance or absence of organ failure $[9,25]$.

We chose a scoping review methodology because initial searches yielded few returns in the target setting. We also chose to take a broad approach to clarify key concepts in delirium detection in cancer settings and identify key concepts and gaps in the evidence base [26]. Our review explores the literature in relation to delirium detection and missed delirium in the inpatient oncology setting, and clinical factors associated with misdiagnosis. The aim of this scoping review is to synthesise knowledge and identify gaps relating to detection tool selection, incidence, prevalence and reversibility of delirium in hospitalised, adult patients with cancer.

\section{Patients and methods}

The target population was admitted, adult, oncology patients in an acute-hospital or comprehensive cancer centre. The research questions were:

1. Which instruments are most commonly used to detect delirium?

2. Which reference standards have been used to measure rates of delirium and compare the performance of delirium screening instruments?

3. What is the incidence and prevalence of delirium in the target setting? and

4. What is the rate of reversibility of delirium in the target setting?

Our search strategy centred on four key domains; delirium, cancer, inpatient oncology, and delirium detection. Full inclusion criteria were: original study, English language, for inclusion the focus of the study must be syndromic delirium e.g. not: confusion, cognitive impairment, acute brain syndrome. The target population is patients with cancer and the setting is adult inpatient oncology, studies not relevant to this population were excluded. Specifically, the target setting was oncology wards in acute hospitals including tertiary referral and cancer centres. Studies of non-oncology ward patients were included if the oncology population could be abstracted from a broader study e.g., hospital-wide point prevalence, subset of cancer patients within an index population of older patients with cancer. Studies set in palliative care populations in a "stand alone" inpatient unit or hospice were only included if the setting was combined oncology and palliative care, for example a comprehensive cancer centre. To meet inclusion a delirium assessment with a validated objective tool, or clinical diagnostic criteria was also required.

Studies were excluded if they were solely conducted in the following settings or populations; haematology or nonsolid haematological malignancy, non-cancer palliative care, perioperative including surgical oncology, or alcohol withdrawal delirium.

The reason for excluding non-solid haematological malignancy was the consideration that illness trajectories and treatment protocols in this population may differ a great deal 
from those of solid cancers, similarly for non-cancer palliative care patients. The exclusion of surgical oncology and peri-operative settings was pragmatic as those patients may be admitted to surgical wards with a different background for staff and potentially different delirium aetiologies. Understanding delirium in these patient cohorts is important and we hope that future work will address areas not included in our review as has been the case in recent multicentre delirium prevalence studies [27, 28].

All authors and an academic liaison-librarian were involved in an iterative process to determine search terms. MEDLINE, CINAHL, PsycINFO, EMBASE and SCOPUS databases were searched. Publication date was limited from 1st of January 1996 to 12th of August 2017. A full list of keywords and Medical Sub-heading (MeSH) is available in Appendix 1.

Independent title, abstract, full-text review and cross check was carried out by MBS and IW, using COVIDENCE [29] software, with conflicts resolved by consensus. Where the same study was reported in more than one manuscript, additional information from related or subsequent publications was included where possible [9, 30-34]. Study heterogeneity was not objectively tested, but the overall lower quality of several included studies and issues with reference standards seemed to suggest meta-analysis would not be meaningful, but sources of bias and generalisability were assessed using the Quality Assessment of Diagnostic Accuracy Studies (QUADAS-2) system.[35] Two authors (MBS and IW) independently piloted the QUADAS-2 and subsequently quality considerations and information synthesis was reviewed by all authors consensus was achieved through discussion.

For the purposes of this study, we defined a delirium reference standard as one which determined diagnostic assignment based on an instrument which used an independent reference-rater evaluation [36]. This last point, although identified in the literature was also arrived at via an iterative process that revealed unclear distinctions between screening tools and case ascertainment or diagnostic criteria upon which case identification was verified among included studies. Examples of reference standards in the basis of these criteria are the World Health Organization (WHO) International Classification of Diseases, 10th Revision (ICD-10) [37] or the American Psychiatric Association Diagnostic and Statistical Manual of Mental Disorders (DSM) [1, 38] criteria, applied by a psychiatrist or consultant physician. On the basis of these criteria, the Confusion Assessment Method (CAM) meets reference standard criteria for case ascertainment, only in studies where reference-rater training in the use of the CAM is explicitly-stated [39]. This follows published recommendations for valid use of the CAM[39] along with diagnostic assignment in delirium research [36]. With regard to detection tool we use the term detection instrument (or tool) to include screening tools or other instruments put forward as standardised methods to identify delirium.

With regard to protocol registration, on inception authors were advised that PROSPERO did not currently accept registrations for scoping reviews and was unable to accept our application for protocol registration. The following is an accurate description of our methodology and further information is available on request. The data that support the findings of this study are available from the corresponding author.

\section{Results}

\section{Search results}

The search date was August 12, 2017. Returns were as follows: Medline (211), EMBASE (684), SCOPUS (97), PsycINFO (52) and CINAHL (47). A total of 805 studies were identified with an additional 91 titles added from hand search. Although most duplicates were removed prior, for pragmatic reasons, final removal of duplicates and screening of abstract and date of publication for hand search returns was held over until full-text review. Hand search consisted of hand search of reference lists from included studies as well as search of authors PDF library using delirium as a title search.

In total, 91 studies were retained for full-text review, and 12 studies remained for data abstraction and synthesis (Fig. 1)

\section{Characteristics of included studies}

Study recruitment periods ranged from 1997 to 2015. Study design of all 12 studies was observational: six were prospective, six were retrospective. Two studies were secondary analyses of data from prior prospective studies. Tables 1 and 2 provide detailed data for the included studies related to the research questions. Table 3 summarises quality considerations according to QUADAS-2 criteria [35]. Table 4 provides a summary

Studies were grouped into three categories on the basis of the clinical setting: (1) inpatient, acute-hospital or comprehensive cancer centre oncology ward; (2) older oncology patients (patients $>65$ years, admitted to acute hospitals under any admitting team, with cancer as the primary diagnosis or co-morbidity); and (3) palliative care ward in acute-hospital or comprehensive cancer centre (APCU). The rationale for this grouping was based in the observation that clinical care for oncology patients occurs largely in one of these three settings, but that from the point of view of research, these settings tend to be studied independently; we also wanted to decrease heterogeneity within subgroups, but 
Fig 1 Flow diagram of literature search. Although most duplicates were removed prior, for pragmatic reasons abstract screening for hand search returns was held over until full text review
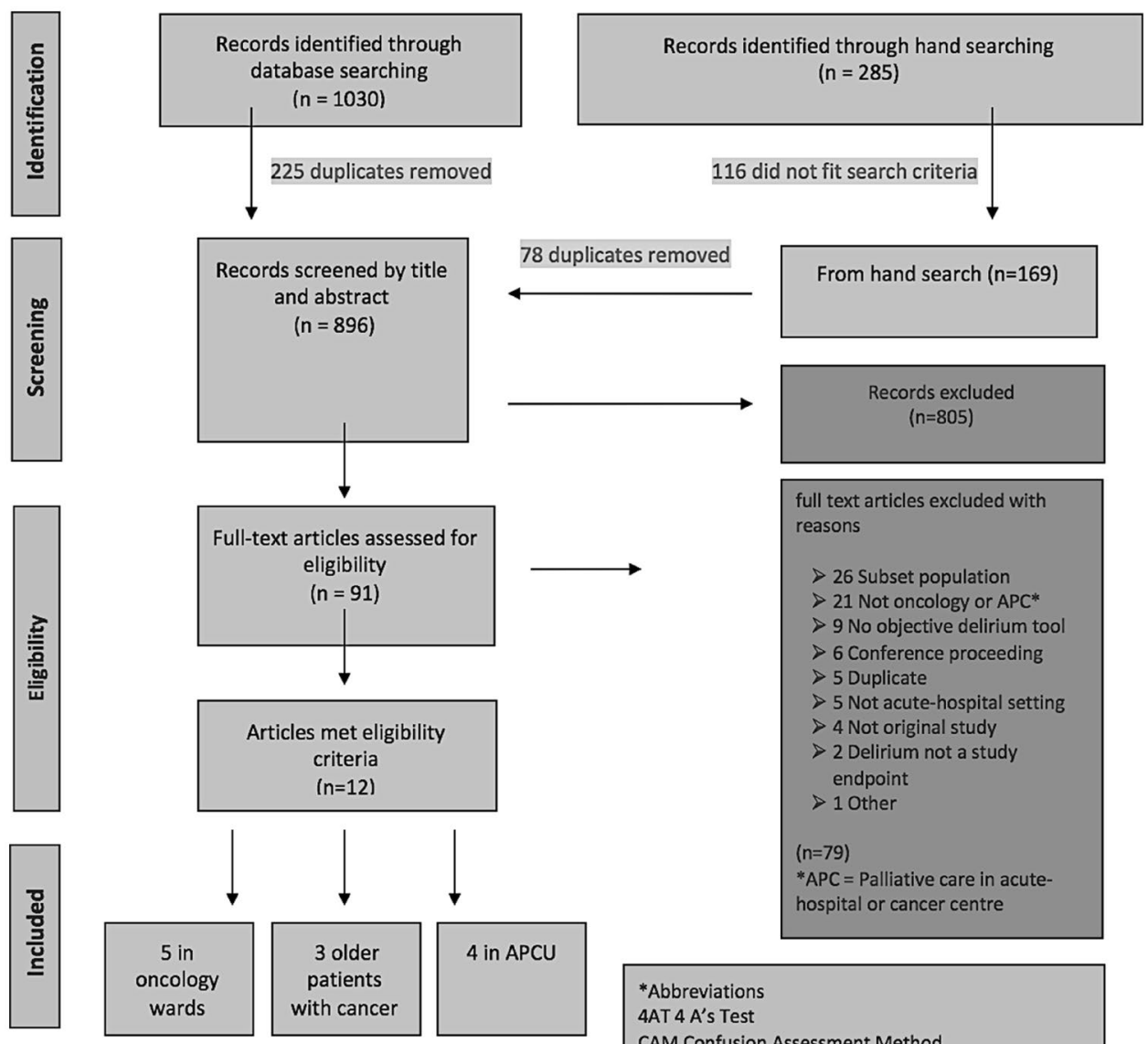

Articles met eligibility criteria ( $n=12$ )
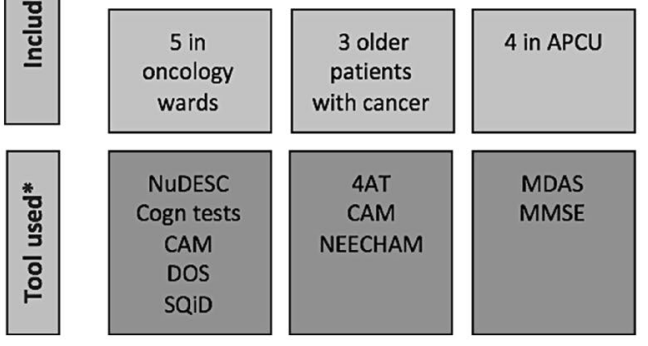

facilitate understanding the use of delirium detection tools across the spectrum of admitted adult patients with cancer to improve care in this cohort.

Five studies [3, 31, 40-42] were in the adult-oncology setting. Three of these approached all patients on nominated days [40-42], while two studies approached all admitted patients $[3,43]$. Three studies were of older cancer patients $[27,32,44]$. One of these was a point prevalence study in which patients over 65 years were recruited from more than 100 hospitals across several regions of Italy during one 24-h period [27]. Unpublished subset data on patients in this last cohort, were provided by the author. (personal communication G Bellelli, October 2017) [45] A further study recruited all patients aged 65 years or older admitted to the general medicine or oncology ward in two Dutch teaching hospitals [44]. The remaining study in older oncology patients, was a secondary analysis of a subset of cancer patients from a previous study, composed of patients from three North American centres [32]. Four studies [9, 46-48] were in an APCU. Three of these
[46-48] were retrospective and based in the same health care facility.

Three studies focused on patients with cancer referred to consultation psychiatry services and reported misdiagnosis of neuropsychiatric conditions, with two studies reporting a missed diagnosis of delirium in $46 \%$, and a further study reporting $63 \%$ missed cases $[11,13,49]$.

\section{Patient recruitment and demographics}

Patient characteristics were described in varying detail: four studies [3, 9, 30, 40] specified histological diagnosis of cancer, and three specified consecutive recruitment [27, $34,46]$. Four studies gave a detailed description of recruitment $[3,44,50]$, and seven provided the number of eligible patients when providing number of participants. Eight studies $[3,9,31,41,42,46-48]$ presented flow diagrams or data accounting for eligible patients not included in recruitment or analysis. 


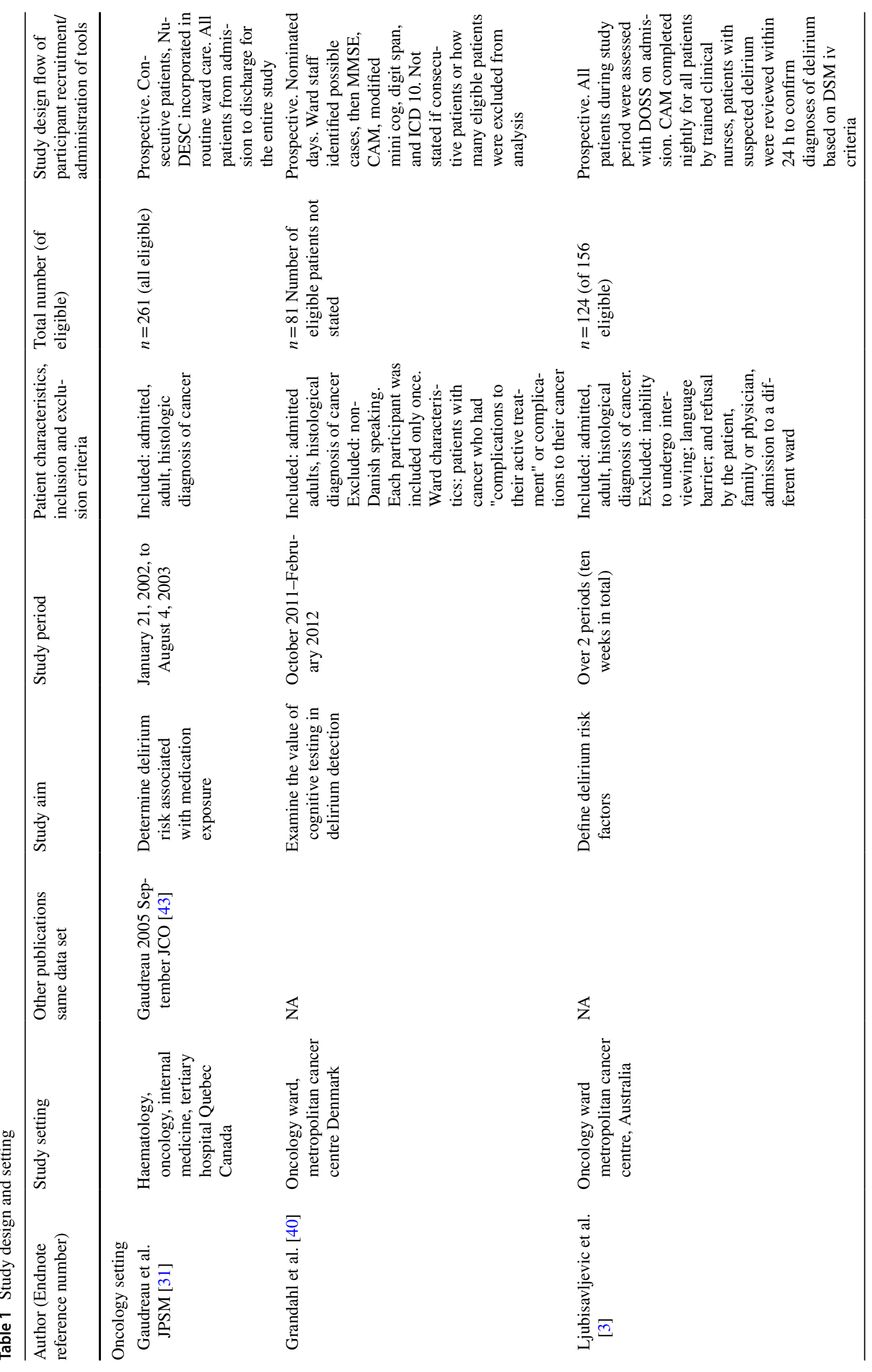




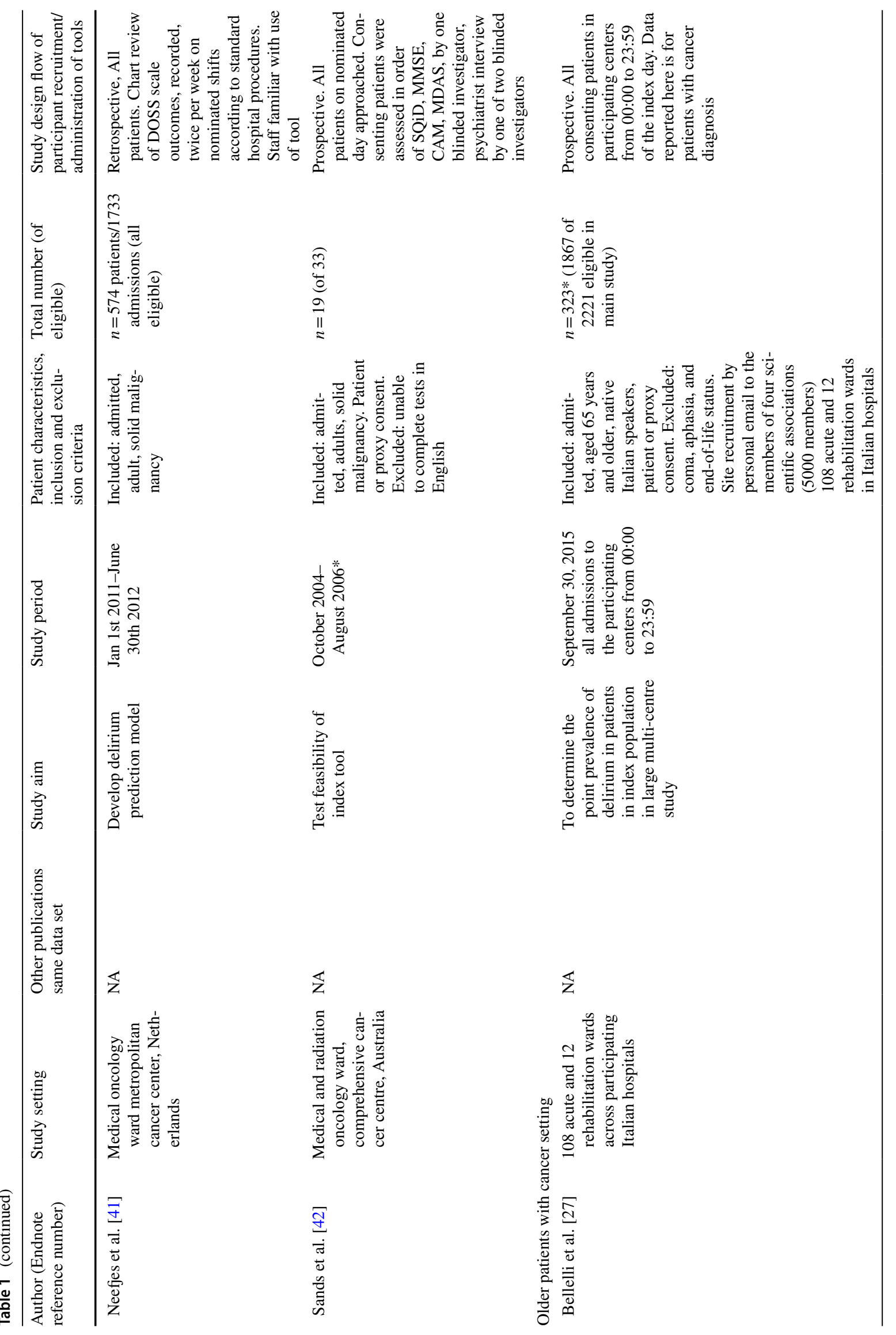




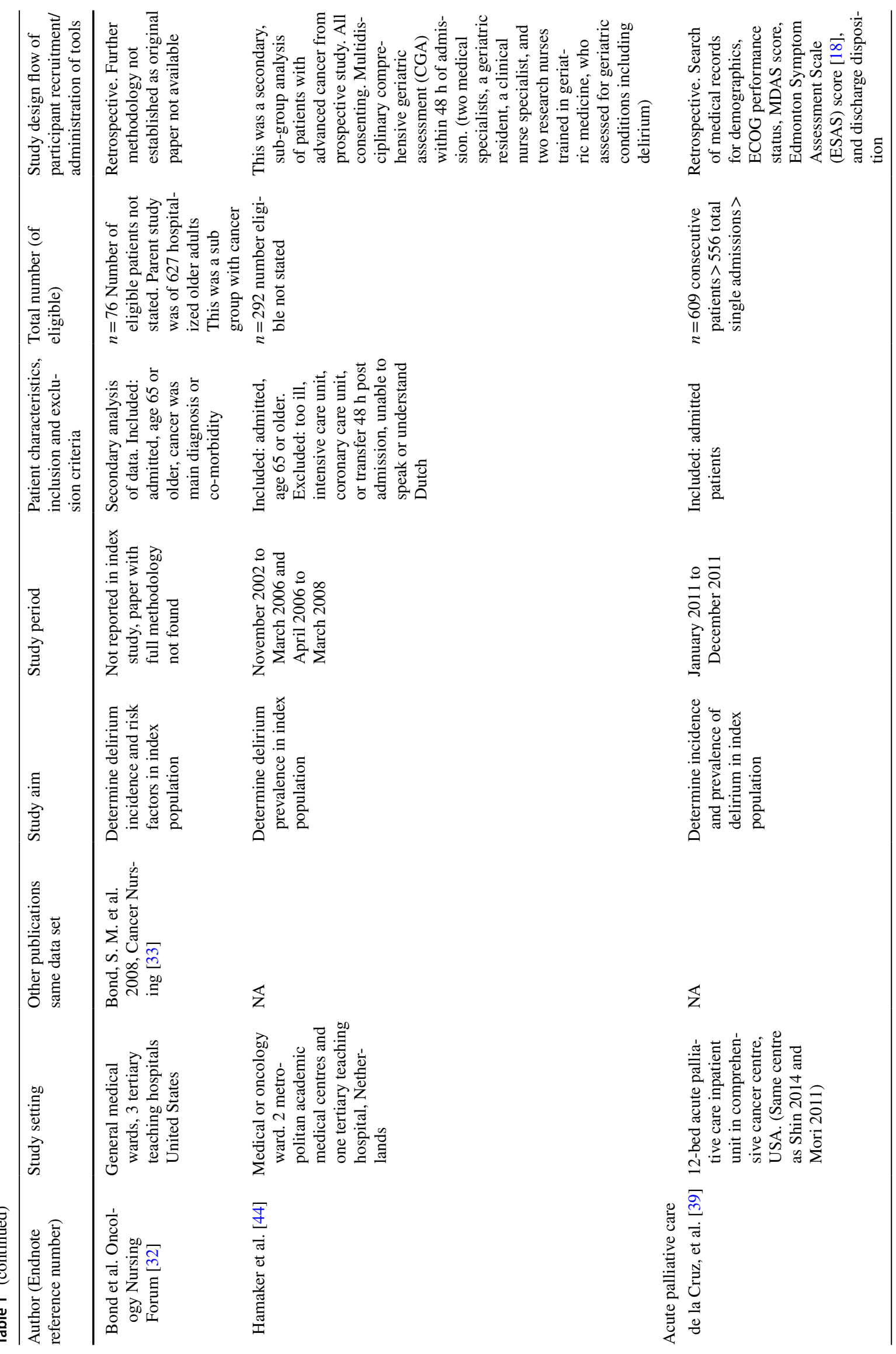




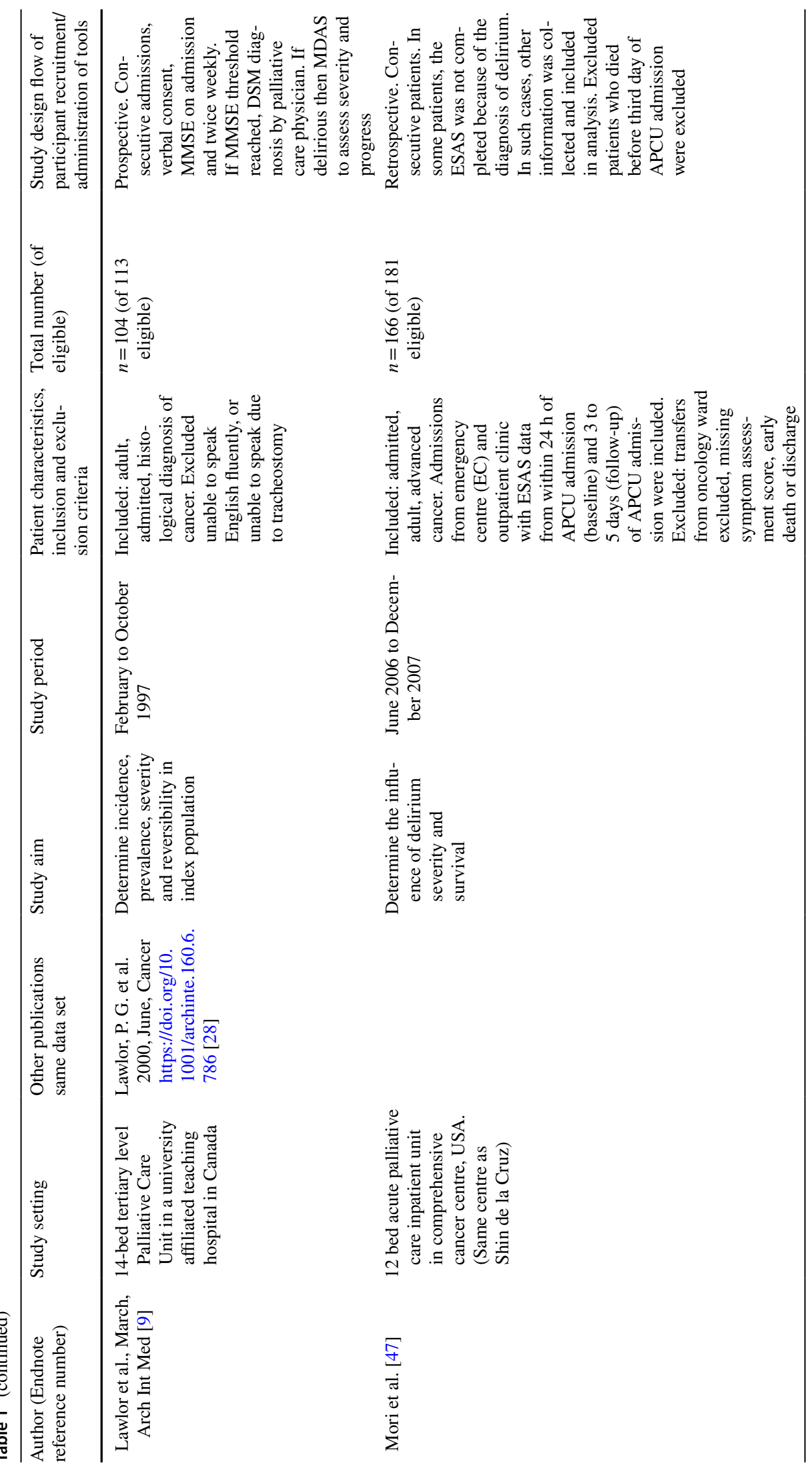




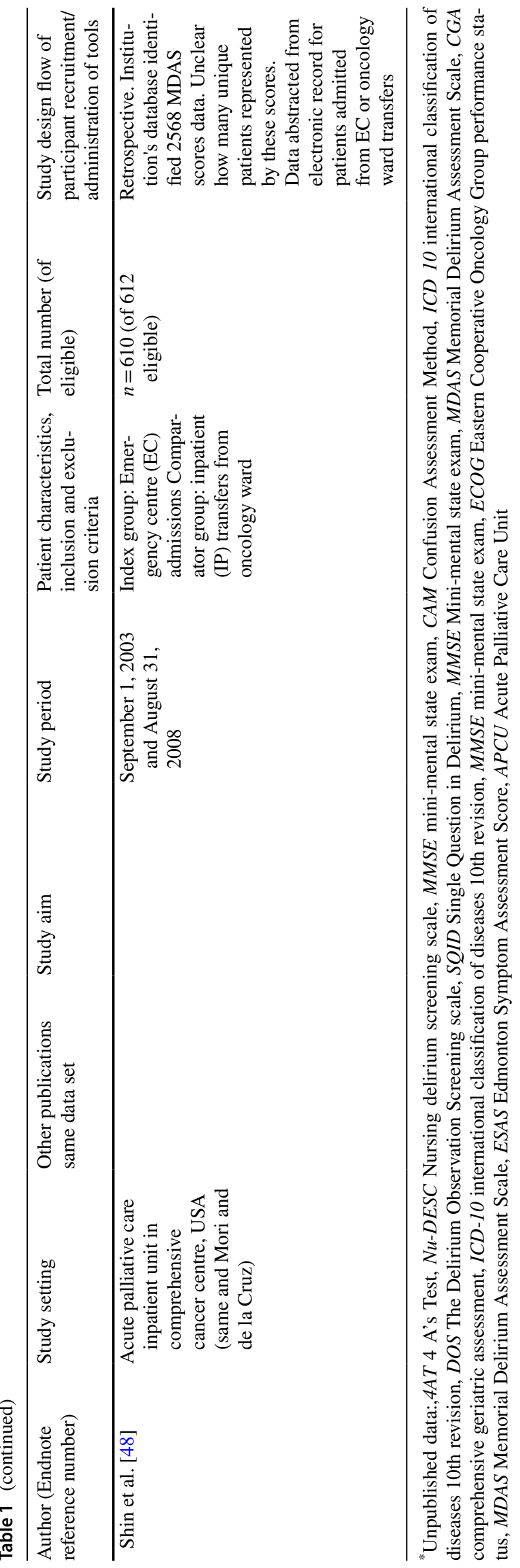

Ten of 12 studies reported primary cancer types. All reported age; the average of the mean age (years) in each setting were as follows: oncology 59, older cancer 78 , and APCU 60. Six studies reported length of hospital stay [3, 32, $41,44,47,48]$; these were reported as mean or median, and ranged from 3 to 9.8 days. Clinical information describing cancer stage, co-morbidity burden, overall illness severity, functional status or vital status at discharge, were not uniformly described. Only one of five studies in the adult oncology setting provided detailed information that described markers of burden of disease [41]. Six of 12 studies across all setting subgroups reported the stage of cancer in terms of metastatic versus loco-regional disease [9, 41-44, 47]. One study reported the number of patients receiving anti-cancer treatment [44].

\section{Scoping questions; data relating to our four research questions}

1. Which instruments are most commonly used to detect delirium?

Of the studies meeting our inclusion criteria, five used previously validated instruments for clinical detection of delirium: Nursing Delirium Screening scale (NuDESC, $n=1$ ); Delirium Observational Screening Scale (DOSS, $n=1$ ); four A's test (4AT, $n=1$ ); Neelon and Champagne (NEECHAM, $n=1$ ); and, Memorial Delirium Assessment Scale (MDAS, $n=4$ ). One study tested a novel delirium screening tool (Single Question in Delirium; SQiD) and one tested cognitive measures (Clock Drawing Test, Mini Cognitive, Digit Span Test) against a reference standard. Six studies included a second delirium detection tool, as presented in Table 2 .

2. Which reference standards have been used to measure rates of delirium?

Four of 12 studies met criteria for a delirium reference standard for case ascertainment [36]. Two studies in the adult oncology setting used diagnostic criteria, namely the ICD 10 [40] (assessor characteristics were not stated), and DSM IV/IVR (assessed by final year psychiatry fellow or psychiatrist) [42]. Two studies used the CAM in a way that met criteria for use as a reference standard, including an account of assessor training [3, 31] .

Seven studies used a screening tool alone as the basis of case ascertainment of delirium: MDAS $(n=4)$; CAM $(n=2)$; DOSS $(n=1)$; 4AT $(n=1)$; NEECHAM $(n=1)$; Nu-DESC $(n=1)$, and one used a battery of tests of cognition $(n=1)$. Neither of these two studies using the CAM as the basis of delirium case ascertainment, specified assessor training $[40,44]$. Of the prospective studies, Bellelli 


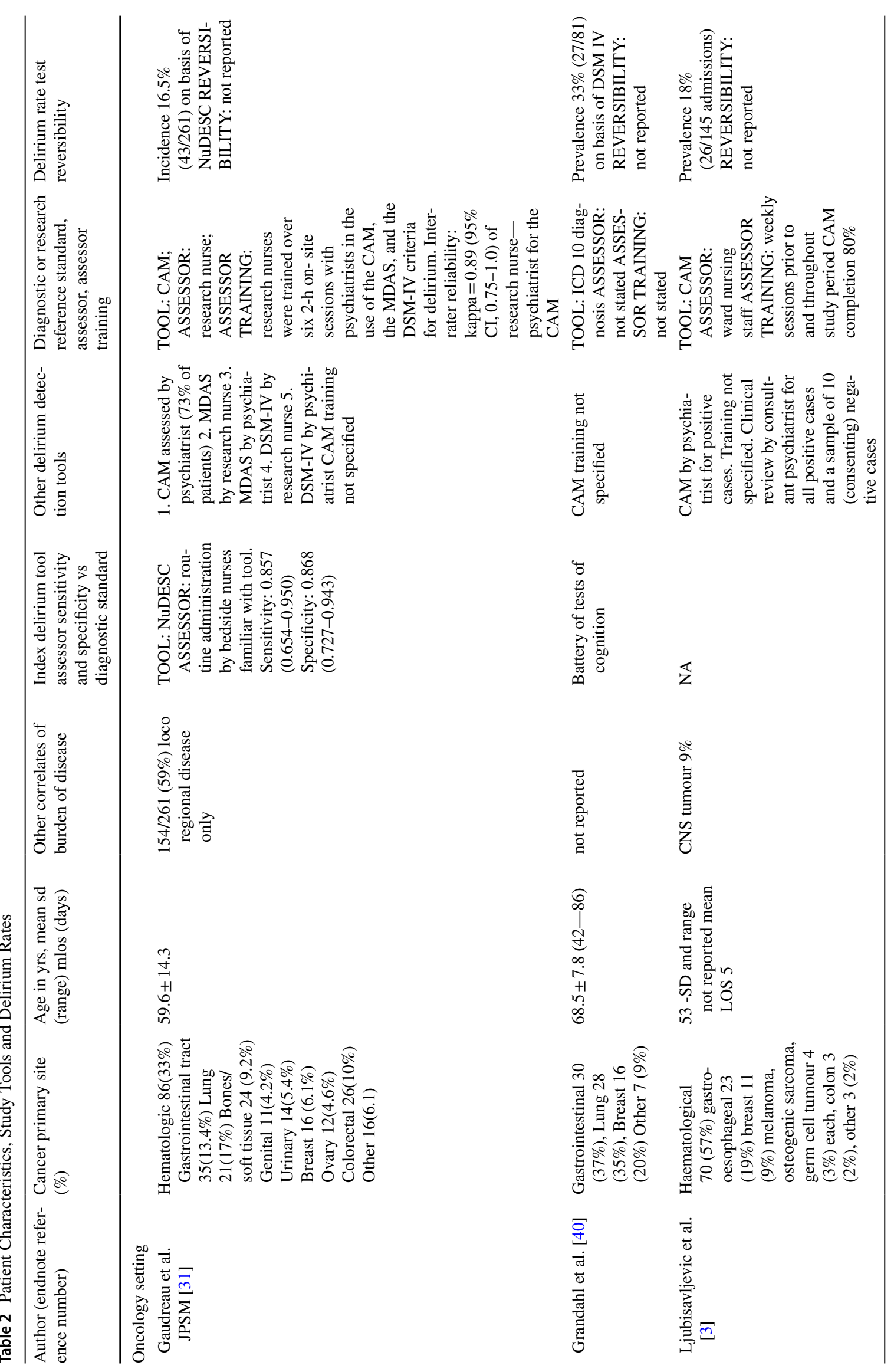




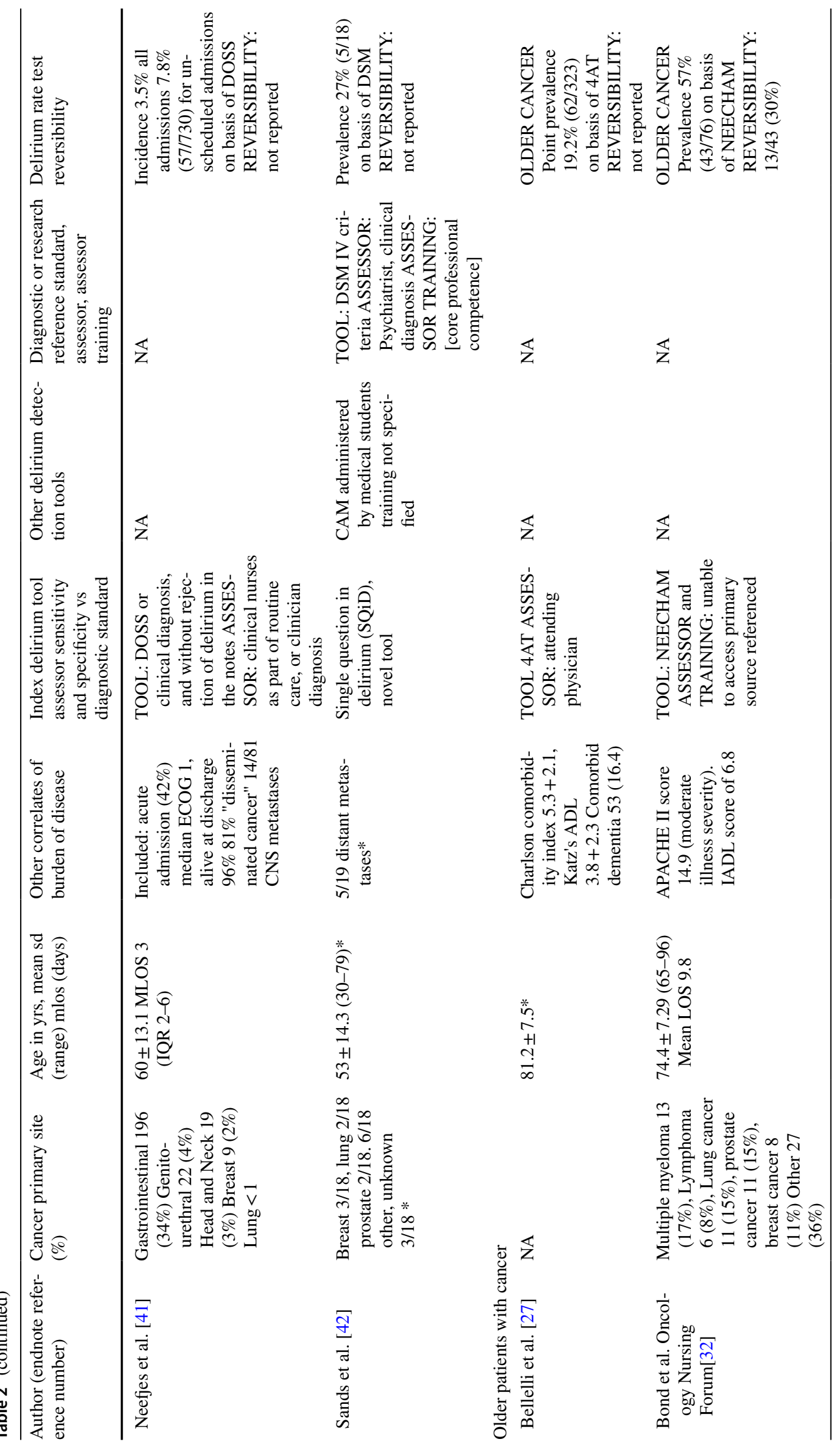




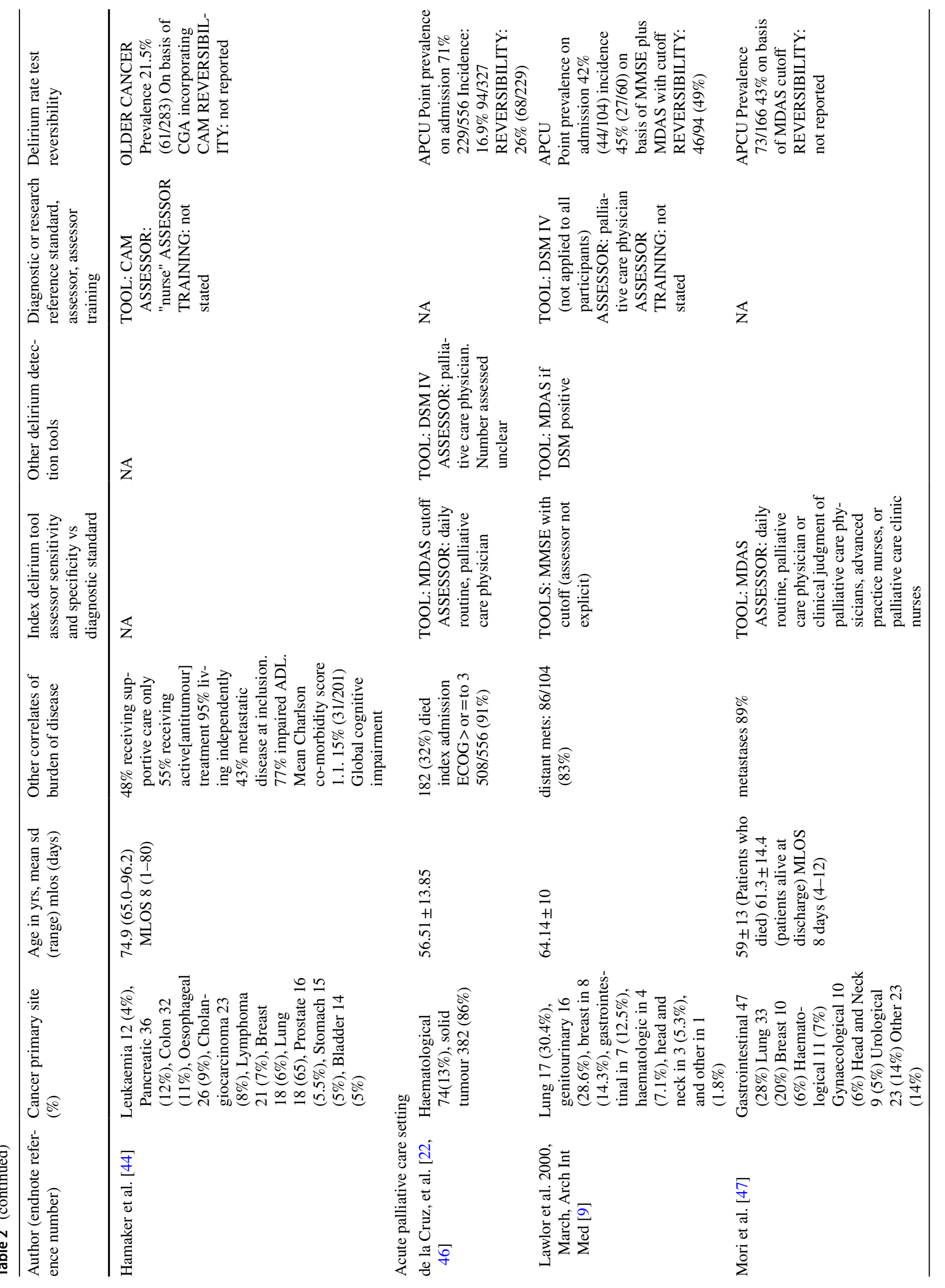




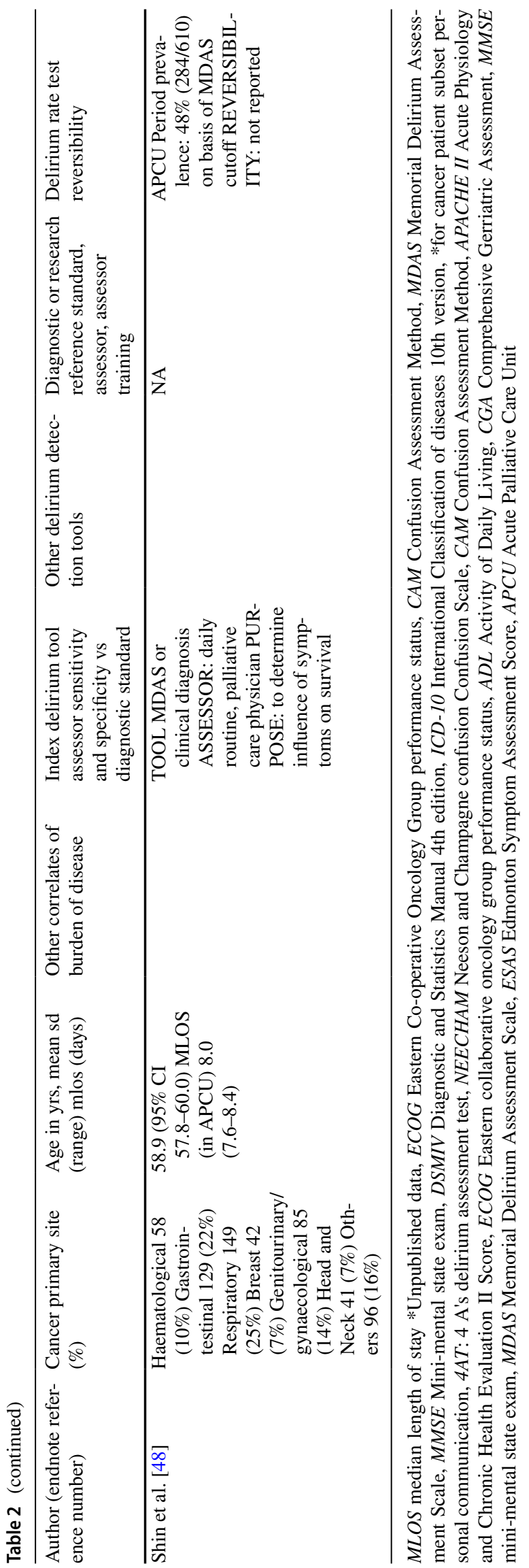

et al. used the $4 \mathrm{AT}$ assessed by the attending physician [27]; Lawlor et al. used DSM IV to confirm participants who had Mini-Mental State Exam (MMSE) scores above a cut-off point on first-line testing [9]; and Gaudreau et al. used the Nu-DESC, applied by trained bedside nurses familiar with this tool [43]. Of the retrospective studies, Neefjes et al. used the DOSS applied by trained bedside nurses familiar with the tool [41], and three studies used a cut-off score on the MDAS to identify delirium cases on chart review [46-48]. Studies comparing MDAS, MMSE, 4AT, NEECHAM tools for detection in clinical practice compared to a reference standard were not identified in our target settings, so it is not possible to ascertain the rate of missed delirium from the available literature.

3. What is the incidence and prevalence of delirium in this setting?

Rates of delirium incidence and prevalence reported by studies in this review are presented in Table 2 . Table 4 presents a summary of tools used and delirium rates established on that basis. Consecutive or non-consecutive recruitment is also reported to aid interpretation of delirium rates.

In the adult oncology setting, Neefjes et al. found a delirium incidence of 3.5 per 100 admissions or 7.8 per 100 of unscheduled admissions [41], and Gaudreau et al. reported an incidence of $16.5 \%$ [43]. Three studies in this sub-setting presented prevalence data; $18 \%$ [3], 27\% [42], and 33\%[40] respectively. In the APCU sub-population, prevalence rates of $42 \%$ [9], 43\%,[47], 48\% [48], and 58\% [46] were found. The three studies of older cancer patients found prevalence rates of $19.2 \%$ [27], 21.5\% [44], and 57\%,[32], respectively. One study in the oncology sub-setting [41], and one in the APCU sub-setting [46], reported the frequency of delirium subtypes: hyperactive 11/52 (21\%) and 61/246 (25\%); hypoactive 20/52 (38\%) and 73/245 (30\%); mixed 18/52 (35\%) and 112/246 (46\%); and not known 3/52 (6\%) and 73/246 (31\%) respectively.

4. What is the rate of reversibility of delirium in this setting?

Delirium reversibility was reported in three studies, two of these were in APCU settings; 46/94 (49\%) [9], 68/229 (26\%) [46], and one in older patients with cancer $13 / 43$ (30\%)[33]. Of four studies reporting reversibility [9, 32, $46,47]$, two [9, 32] did not explicitly state how reversibility was defined; one used the MDAS or clinical documentation to determine delirium reversibility and another used a MDAS cut off score at day five. Although one of these[47] referenced a predating publication, this could not be found. The other three used MDAS scores collected as 
Table 3 Quality assessment using QUADAS tool

\begin{tabular}{|c|c|c|c|c|c|c|c|}
\hline $\begin{array}{l}\text { Author (End- } \\
\text { note reference } \\
\text { number) }\end{array}$ & $\begin{array}{l}\text { Risk of bias } \\
\text { patient selection }\end{array}$ & $\begin{array}{l}\text { Risk of bias } \\
\text { index test }\end{array}$ & $\begin{array}{l}\text { Risk of bias ref- } \\
\text { erence standard }\end{array}$ & $\begin{array}{l}\text { Risk of bias } \\
\text { flow and timing }\end{array}$ & $\begin{array}{l}\text { Generalisability } \\
\text { patient selection }\end{array}$ & $\begin{array}{l}\text { Generalisability } \\
\text { index test }\end{array}$ & $\begin{array}{l}\text { Generalis- } \\
\text { ability reference } \\
\text { standard }\end{array}$ \\
\hline \multicolumn{8}{|l|}{ Oncology setting } \\
\hline $\begin{array}{c}\text { Gaudreau et al. } \\
\text { April [31] }\end{array}$ & Low risk & Low risk & Low risk & Low risk & $\begin{array}{l}\text { Intermediate } \\
\text { risk }\end{array}$ & Low risk & Low risk \\
\hline $\begin{array}{l}\text { Grandahl et al. } \\
\text { [40] }\end{array}$ & Low risk & $\begin{array}{l}\text { Intermediate } \\
\text { risk }\end{array}$ & $\begin{array}{l}\text { Intermediate } \\
\text { risk }\end{array}$ & $\begin{array}{l}\text { Intermediate } \\
\text { risk }\end{array}$ & $\begin{array}{l}\text { Intermediate } \\
\text { risk }\end{array}$ & Low risk & Low risk \\
\hline $\begin{array}{l}\text { Ljubisavljevic } \\
\text { et al. [3] }\end{array}$ & Low risk & Low risk & Low risk & Low risk & Low risk & Low risk & Low risk \\
\hline $\begin{array}{l}\text { Neefjes et al. } \\
\text { [41] }\end{array}$ & Low risk & Low risk & Not used & $\begin{array}{l}\text { Intermediate } \\
\text { risk }\end{array}$ & $\begin{array}{l}\text { Intermediate } \\
\text { risk }\end{array}$ & Low risk & Not used \\
\hline $\begin{array}{l}\text { Sands et al. } \\
\text { [42] }\end{array}$ & Low risk & $\begin{array}{l}\text { Intermediate } \\
\text { risk }\end{array}$ & Low risk & Low risk & $\begin{array}{l}\text { Intermediate } \\
\text { risk }\end{array}$ & Low risk & Low risk \\
\hline \multicolumn{8}{|c|}{ Older patients with cancer setting } \\
\hline $\begin{array}{l}\text { Bellelli et al. } \\
\text { [27] }\end{array}$ & Low risk & Low risk & Low risk & Low risk & $\begin{array}{l}\text { Intermediate } \\
\text { risk (for can- } \\
\text { cer subset) }\end{array}$ & Low risk & Low risk \\
\hline $\begin{array}{l}\text { Hamaker et al. } \\
\text { [44] }\end{array}$ & Low risk & Not used & Low risk & Low risk & Low risk & Low risk & Low risk \\
\hline $\begin{array}{l}\text { Bond et al. } \\
\text { [32] }\end{array}$ & \multicolumn{7}{|c|}{ Insufficient information to assess } \\
\hline \multicolumn{8}{|c|}{ Acute palliative care setting } \\
\hline $\begin{array}{l}\text { de la Cruz, } \\
\text { et al. }[22,46]\end{array}$ & $\begin{array}{l}\text { Intermediate } \\
\text { risk }\end{array}$ & Higher risk & $\begin{array}{l}\text { Intermediate } \\
\text { risk }\end{array}$ & $\begin{array}{l}\text { Intermediate } \\
\text { risk }\end{array}$ & $\begin{array}{l}\text { Intermediate } \\
\text { risk }\end{array}$ & $\begin{array}{l}\text { Intermediate } \\
\text { risk }\end{array}$ & Higher risk \\
\hline $\begin{array}{l}\text { Lawlor et al. } \\
\text { [9] }\end{array}$ & Low risk & Higher risk & Low risk & Higher risk & Low risk & Higher risk & Higher risk \\
\hline $\begin{array}{l}\text { Mori et al. } \\
2011 \text { [47] }\end{array}$ & $\begin{array}{l}\text { Intermediate } \\
\text { risk }\end{array}$ & Higher risk & Higher risk & $\begin{array}{l}\text { Intermediate } \\
\text { risk }\end{array}$ & $\begin{array}{l}\text { Intermediate } \\
\text { risk }\end{array}$ & Higher risk & Higher risk \\
\hline Shin et al. [48] & $\begin{array}{l}\text { Intermediate } \\
\text { risk }\end{array}$ & Higher risk & Higher risk & $\begin{array}{l}\text { Intermediate } \\
\text { risk }\end{array}$ & $\begin{array}{l}\text { Intermediate } \\
\text { risk }\end{array}$ & $\begin{array}{l}\text { Intermediate } \\
\text { risk }\end{array}$ & Higher risk \\
\hline
\end{tabular}

Total number of studies in categories: Study Setting: Oncology (5), older patients with cancer (3), acute palliative care (4). Diagnostic reference standards (2): DSM Diagnostic and Statistics Manual (various editions ICD-10 International Classification of Diseases (10th version) and CAM by trained operator (1). Tools used for delirium detection: MDAS (4), CAM (3), DOSS (1), Cognition testing (1), 4AT (1), NEECHAM (1), NuDESCC (1) Note: (total greater than number of studies as one study used two methods)

part of routine clinical care to define reversibility. Bond et al. found that patients with fewer precipitating factors were more likely to have a resolution but found only prior cognitive impairment to be negatively associated with delirium reversal [33].

In the Lawlor study delirium associated with opioids and non-opioid psychoactive medication and dehydration were more likely to be reversed while non-reversed delirium was more common when associated with a respiratory infection, pulmonary cancer and metabolic causes [9].

\section{Discussion}

We identified significant knowledge gaps regarding epidemiological characteristics of delirium in oncology inpatients. A variety of delirium screening tools were identified, but few studies used accepted diagnostic or reference standards for case ascertainment. Sources of bias included study design and generalisability. A small number of eligible studies reported reversibility of delirium.

Delirium is a multifactorial syndrome. The relationship of delirium risk with demographic factors such as age and clinical factors (e.g. cancer diagnosis), is complex. One of the studies in the older cancer sub-group provided comparative figures of delirium point prevalence in cancer and non-cancer patients; $19.2 \%(n=323)$ for patients with cancer compared to $23.9 \%(n=1544)$ of the patients with no cancer diagnosis $(p=0.06)$ [27]. Within the included studies, but also in non-cancer settings reported elsewhere, multiple delirium risk factors such as co-morbidities, presence of physiological disturbance and medication burden are factors that seem to be constants in understanding overall delirium risk. [7, 9, 13, 41, 43, 51-59]

Delirium screening tools have been developed, and validated, predominantly in older, hospitalised patients [60-64]. 


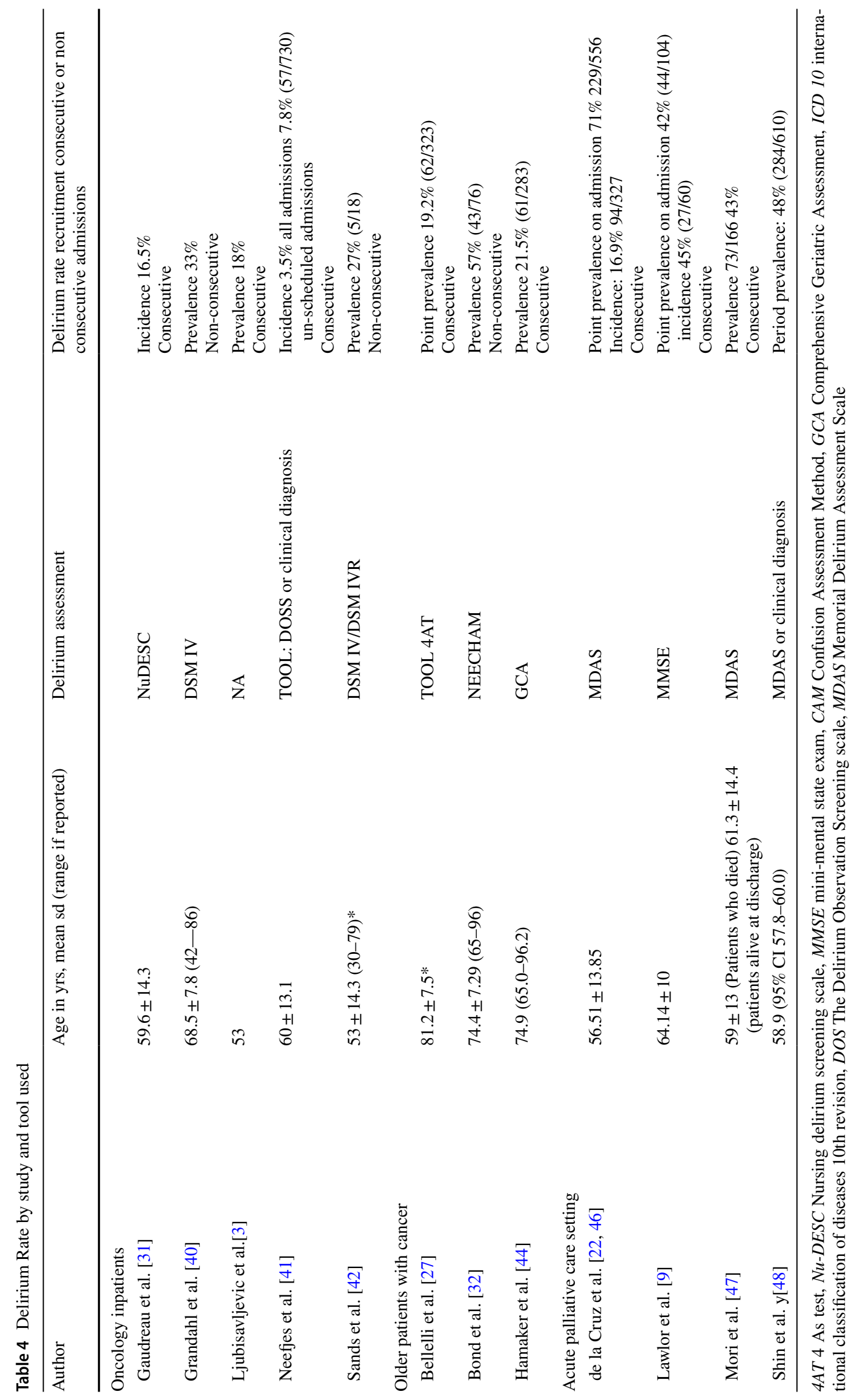


The tools for which psychometric properties have been assessed in cancer in-patients in the acute setting, are the Nu-DESC and MDAS [31, 34, 65]. There is a clear rationale for use of the Nu-DESC as a delirium screening tool [31]. The 4AT has been tested in "stand-alone" palliative care, inpatient settings[66] and for older adults admitted to hospital [50, 67, 68]. The MMSE can be used to screen for cognitive impairment it has been found to have poor performance as a bedside tool for identifying delirium $[69,70]$.

The Confusion Assessment Method [36] (CAM) has several versions [67], and has well-established psychometric properties [61]. In the main, studies have supported the use of the CAM for delirium screening in research settings providing there is strict adherence to operator training, however, one study suggests even in the context of strict adherence to CAM training, sensitivity of the CAM may not be sustained [68]. Our review found that most studies using the CAM for case ascertainment did not describe the training staff underwent $[3,37,39,41]$ and one, described difficulty in attaining adequate training in a clinically embedded research context $[42,71]$.

Four of the twelve included studies used the MDAS as a basis for case confirmation of delirium [9, 46-48]. The MDAS was designed specifically to rate delirium severity[65], it has face validity and uptake, further formal validation studies for its use as a delirium screening tool would build on the existing psychometric data and help to reinforce the attributes of the tools [64]. One perspective looks at the balance between the positive features of usability of the MDAS compared with some other tools, and the effect of the breakdown and operationalisation of delirium features within the MDAS which does not support the syndromic nature of delirium diagnosis in terms of coexisting core features. Although it identifies delirium symptoms, regardless of the cut-off score specified to identify delirium, the MDAS risks false positive results, as patients with delirium symptoms who do not fit the core diagnostic criteria for syndromic delirium as characterised by coexistent core features may be labelled case positive. Several studies in this review used the MDAS alone for case ascertainment, which may bias reported detection rates [48, 64].

Clinical operationalisation appeared to be the major driver of choice of delirium screening tools. Delirium diagnosis is complex, multidimensional and not intuitive for bedside staff. DSM 5 criteria require 5 characteristics and so while screening tools may gain in usability through operationalisation they lose precise application of the necessarily coexistent core features that define delirium. In the research setting, we found clear demarcation between the index tool and the chosen reference standard was not always evident. A blurring of the distinction between screening tools and diagnostic reference standards used for case confirmation for validation purposes was found. More specifically where references standards were other than DSM or ICD based reporting of reference-rater training was at times lacking. The importance of tool selection to fit the intended purpose is an important finding of our review.

In APCUs delirium rates were higher than in oncology inpatients but given methodological constraints in studies within this setting, results may not be representative. The use of the MDAS may have contributed to inflated delirium rates reflecting the way the tool is operationalised. In the older cancer patient cohorts, differences in delirium incidence and prevalence might be accounted for by study heterogeneity and patient recruitment. This is an important issue for future work, as establishing delirium incidence and prevalence in inpatient oncology settings is an important step in management. Better understanding of how to use available tools will improve management and inform education initiatives in this setting.

Criteria for delirium reversal were inadequately defined in studies, making it difficult to compare delirium reversibility across studies. These data may be further constrained by retrospective methodology, the absence of a diagnostic reference standard, or study flow reliant on clinical documentation. Ascertainment of delirium reversibility requires prospective, longitudinal study design, use of a robust diagnostic standard and explicit definition of delirium reversal. Assessment of delirium reversibility is an important issue for consideration in the design of future studies.

Patient selection, choice of the delirium screening tool and the choice of the diagnostic reference standard, were all identified as a source of bias on QUADAS-2 criteria [35]. Recruitment flow was also an important consideration. For example, patient selection methods at times risked exclusion of potentially delirious patients due to retrospective design, convenience sampling, and ascertainment bias.

Adherence to consensus recommendations for reporting patient characteristics and wherever possible the use of assessment tools and delirium reference standards will improve epidemiological studies of delirium in this setting $[36,72,73]$.

Limitations to our review include those related to the methodology of the original studies as well as a limitation to the English language. The search was limited to publications between 1996 and 2017. The discussion has aimed to identify recent updates in the area, again these are largely limited to aged care or stand-alone settings, with one systematic review of delirium in palliative care fining an incidence of 9-57\% across hospital palliative care consultative services, with a majority of patients having cancer diagnoses [20]. a further systematic review, again in the palliative care setting, identified 14 delirium detection tools and heterogeneity of methods [23], Important questions for future work include which tools translate 
well to inpatient oncology from aged care and stand-alone inpatient palliative care settings, which tools are most suitable for patients, carers and staff, and which reference standards are most appropriate. Requirements for clinical and research uses of detection tools will differ according to purpose, however establishing methodical approaches to the detection of delirium in either setting is a prerequisite to determining the incidence, prevalence and reversibility of delirium for oncology inpatients. Maintaining a clear accountability for the validation and purpose of the tool, and its psychometric characteristics when applying it to clinical screening/detection is critical as is the requirement in research uses to select a reference standard with established reference-rater methodology, is extremely important.

Choosing a tool for delirium detection in the clinical oncology setting will vary according to operational issues such as staff training and preference, however, it is important that tools are fit for purpose, and where possible, have been validated in the same clinical setting. While patient profiles may be similar across palliative care, aged care and some oncology inpatient settings, staff competencies will be more specifically related to setting. Delirium detection and diagnosis must be a core competency for clinical teams in acute settings, however, operational characteristics may render a tool selection may vary according to operational setting, the exact tool chosen is not as important as the review of characteristics that makes it fit for purpose/setting.

Our review, found gaps in the validation of tools in for use in oncology inpatients. At present extrapolation from findings in other acute hospital settings, such as aged care, may help support a more robust selection for this population for the time being. As further validation occurs in acute oncology settings the evidence base for selection tools to detect the presence or resolution of delirium in this clinical setting should improve.

The knowledge gaps identified to generate new hypotheses for future investigation. We recommend the optimal description of patient characteristics, selection of delirium detection tools appropriate to the setting, use of reproducible methods of patient selection and diagnostic assignment using a reference standard with appropriate reference rater methodology. Our results indicate that a determination of the incidence, prevalence, and reversibility of delirium in the inpatient cancer population is both lacking and overdue. Addressing these knowledge gaps will help to provide a more robust evidence base to inform ongoing efforts for effective prevention, detection and management of delirium in the inpatient oncology setting.

Supplementary Information The online version contains supplementary material available at https://doi.org/10.1007/s41999-021-00586-1.
Acknowledgements The authors are grateful for assistance from Elaine Tam, Academic Liaison Librarian, Medicine and Health, Health Sciences, University Library, University of Sydney in the selection of databases for inclusion as well as keywords and search iterations.

Author contributions This review was conducted as part of research undertaken for a Master of Philosophy at the University of Sydney completed in 2019 by the first author which is available at https:// ses.library.usyd.edu.au/handle/2123/18948. All authors contributed to development of the Bodleian question, analysis, and final manuscript for this scoping review. Assistance from Mrs Elaine Tam Sydney University Library is gratefully acknowledged. Data abstraction and analysis was performed by Dr. MBS and Dr. IW and reviewed by all authors.

Funding Not applicable.

\section{Declarations}

Conflict of interest No authors have conflicting/competing interest.

Availability of data and material Upon request.

Code availability Not applicable.

Ethics approval Not applicable.

Consent to participate Not applicable.

Consent for publication All authors give consent.

Open Access This article is licensed under a Creative Commons Attribution 4.0 International License, which permits use, sharing, adaptation, distribution and reproduction in any medium or format, as long as you give appropriate credit to the original author(s) and the source, provide a link to the Creative Commons licence, and indicate if changes were made. The images or other third party material in this article are included in the article's Creative Commons licence, unless indicated otherwise in a credit line to the material. If material is not included in the article's Creative Commons licence and your intended use is not permitted by statutory regulation or exceeds the permitted use, you will need to obtain permission directly from the copyright holder. To view a copy of this licence, visit http://creativecommons.org/licenses/by/4.0/.

\section{References}

1. American Psychiatric Association (2013) Diagnostic and statistical manual of mental disorders (DSM-5®). American Psychiatric Pub.

2. The European Delirium Association and The American Delirium Society (2014) The DSM-5 criteria, level of arousal and delirium diagnosis: inclusiveness is safer. BMC Med 12(1):141

3. Ljubisavljevic V, Kelly B (2003) Risk factors for development of delirium among oncology patients. Gen Hosp Psychiatry 25(5):345-352

4. Dharmarajan K et al (2017) Pathway from delirium to death: potential in-hospital mediators of excess mortality. J Am Geriatr Soc 65(5):1026-1033

5. Breitbart W, Gibson C, Tremblay A (2002) The delirium experience: delirium recall and delirium-related distress in hospitalized patients with cancer, their spouses/caregivers, and their nurses. Psychosomatics 43(3):183-194 
6. Cohen MZ et al (2009) Delirium in advanced cancer leading to distress in patients and family caregivers. J Palliat Care 25(3):164-171

7. Inouye SK et al (1999) A multicomponent intervention to prevent delirium in hospitalized older patients. N Engl J Med 340(9):669-676

8. Ogawa A et al (2019) Quality of care in hospitalized cancer patients before and after implementation of a systematic prevention program for delirium: the DELTA exploratory trial. Support Care Cancer 27(2):557-565

9. Lawlor PG et al (2000) Occurrence, causes, and outcome of delirium in patients with advanced cancer: a prospective study. Arch Intern Med 160(6):786-794

10. Inouye SK et al (2001) Nurses' recognition of delirium and its symptoms: comparison of nurse and researcher ratings. Arch Intern Med 161(20):2467-2473

11. Swigart SE et al (2008) Misdiagnosed delirium in patient referrals to a university-based hospital psychiatry department. Psychosomatics 49(2): 104-108

12. Bellelli $\mathrm{G}$ et al (2015) Under-detection of delirium and impact of neurocognitive deficits on in-hospital mortality among acute geriatric and medical wards. Eur J Intern Med 26(9):696-704

13. Kishi $Y$ et al (2007) Delirium: patient characteristics that predict a missed diagnosis at psychiatric consultation. Gen Hosp Psychiatry 29(5):442-445

14. Hempenius L et al (2016) Long term outcomes of a geriatric liaison intervention in frail elderly cancer patients. PLoS ONE 11(2): 0143364

15. Bush SH et al (2018) Delirium in adult cancer patients: ESMO clinical practice guidelines. Ann Oncol 29:iv143-iv165

16. Spiller JA, Keen JC (2006) Hypoactive delirium: assessing the extent of the problem for inpatient specialist palliative care. Palliat Med 20(1):17-23

17. Hey J et al (2013) The detection, documentation and management of delirium in 3 palliative care settings. Psychooncology 22:18-19

18. Hosie A et al (2013) Delirium prevalence, incidence, and implications for screening in specialist palliative care inpatient settings: a systematic review. Palliat Med 27(6):486-498

19. Cobb JL et al (2000) Delirium in patients with cancer at the end of life. Cancer Pract 8(4):172-177

20. Watt CL et al (2019) The incidence and prevalence of delirium across palliative care settings: a systematic review. Palliat Med 33(8):865-877

21. Barnes J, Kite S, Kumar M (2010) The recognition and documentation of delirium in hospital palliative care inpatients. Palliat Support Care 8(2):133-136

22. de la Cruz M et al (2015) The frequency of missed delirium in patients referred to palliative care in a comprehensive cancer center. Support Care Cancer 23(8):2427-2433

23. Watt CL et al (2021) Delirium screening tools validated in the context of palliative care: a systematic review. Palliat Med 35(4):683-696

24. Gagnon P et al (2000) Delirium in terminal cancer: a prospective study using daily screening, early diagnosis, and continuous monitoring. J Pain Symptom Manag 19(6):412-426

25. Leonard $\mathrm{M}$ et al (2008) Reversibility of delirium in terminally ill patients and predictors of mortality. Palliat Med 22(7):848-854

26. Aromataris E, MZe (2017) Joanna Briggs Institute reviewer's manual. The Joanna Briggs Institute

27. Bellelli G et al (2016) "Delirium Day": a nationwide point prevalence study of delirium in older hospitalized patients using an easy standardized diagnostic tool. BMC Med 14:106

28. Shenkin SD et al (2019) Delirium detection in older acute medical inpatients: a multicentre prospective comparative diagnostic test accuracy study of the 4AT and the confusion assessment method. BMC Med 17(1):1-14

29. Veritas Health Innovation, M., Australia (2017) Covidence systematic review software. www.covidence.org. Cited 2017

30. Gaudreau JD et al (2005) Association between psychoactive medications and delirium in hospitalized patients: a critical review. Psychosomatics 46(4):302-316

31. Gaudreau JD et al (2005) Fast, systematic, and continuous delirium assessment in hospitalized patients: the nursing delirium screening scale. J Pain Symptom Manag 29(4):368-375

32. Bond SM, Neelon VJ, Belyea MJ (2006) Delirium in hospitalized older patients with cancer. Oncol Nurs Forum 33(6):1075-1083

33. Bond SM, Neelon VJ (2008) Delirium resolution in hospitalized older patients with cancer. Cancer Nurs 31(6):444-451

34. Lawlor PG et al (2000) Clinical utility, factor analysis, and further validation of the memorial delirium assessment scale in patients with advanced cancer: Assessing delirium in advanced cancer. Cancer 88(12):2859-2867

35. Whiting PF et al (2011) QUADAS-2: a revised tool for the quality assessment of diagnostic accuracy studies. Ann Intern Med 155(8):529-536

36. Neufeld KJ et al (2014) Delirium diagnosis methodology used in research: a survey-based study. Am J Geriatr Psychiatry 22(12):1513-1521

37. World Health Organization. International Classification of Diseases (ICD) information sheet. http://www.who.int/classifications/ icd/factsheet/en/index.html. Cited Jan 2013

38. Castillo RJ, Sattler D, Shabatay V, Kramer G, American Psychiatric Association (2000) Diagnostic and statistical manual of mental disorders (DSM-IV-TR)

39. Inouye SK et al (1990) Clarifying confusion: the confusion assessment method. A new method for detection of delirium. Ann Intern Med 113(12):941-948

40. Grandahl MG et al (2016) Prevalence of delirium among patients at a cancer ward: clinical risk factors and prediction by bedside cognitive tests. Nord J Psychiatry 70(6):413-417

41. Neefjes ECW et al (2017) Identification of patients with cancer with a high risk to develop delirium. Cancer Med

42. Sands MB et al (2010) Single question in delirium (SQiD): testing its efficacy against psychiatrist interview, the confusion assessment method and the memorial delirium assessment scale. Palliat Med 24(6):561-565

43. Gaudreau JD et al (2005) Psychoactive medications and risk of delirium in hospitalized cancer patients. J Clin Oncol 23(27):6712-6718

44. Hamaker ME et al (2011) The value of a comprehensive geriatric assessment for patient care in acutely hospitalized older patients with cancer. Oncologist 16(10):1403-1412

45. Bellelli G (2017) Prevalence of delirium in cancer patient sub-set of delirium day study. In: Sands MB (Ed.)

46. de la Cruz $\mathrm{M}$ et al (2015) The frequency, characteristics, and outcomes among cancer patients with delirium admitted to an acute palliative care unit. Oncologist 20(12):1425-1431

47. Mori $\mathrm{M}$ et al (2011) Changes in symptoms and inpatient mortality: a study in advanced cancer patients admitted to an acute palliative care unit in a comprehensive cancer center. J Palliat Med 14(9):1034-1041

48. Shin SH et al (2014) Characteristics and outcomes of patients admitted to the acute palliative care unit from the emergency center. J Pain Symptom Manag 47(6):1028-1034

49. Armstrong SC, Cozza KL, Watanabe KS (1997) The misdiagnosis of delirium. Psychosomatics 38(5):433-439

50. Bellelli G et al (2014) Validation of the 4AT, a new instrument for rapid delirium screening: a study in 234 hospitalised older people. Age Ageing 43(4):496-502 
51. Elie M et al (2000) Prevalence and detection of delirium in elderly emergency department patients. CMAJ 163(8):977-981

52. Morrison C (2003) Identification and management of delirium in the critically ill patient with cancer. AACN Clin Issues 14(1):92-111

53. Gaudreau JD et al (2005) Impact on delirium detection of using a sensitive instrument integrated into clinical practice. Gen Hosp Psychiatry 27(3):194-199

54. Gaudreau JD et al (2007) Opioid medications and longitudinal risk of delirium in hospitalized cancer patients. Cancer 109(11):2365-2373

55. Agar M, Lawlor P (2008) Delirium in cancer patients: a focus on treatment-induced psychopathology. Curr Opin Oncol 20(4):360-366

56. Uchida $\mathrm{M}$ et al (2012) Prevalence, associated factors and course of delirium in advanced cancer patients. Asia Pac J Clin Oncol 8:177

57. Barron EA, Holmes J (2013) Delirium within the emergency care setting, occurrence and detection: a systematic review. Emerg Med J 30(4):263-268

58. Alexander K, Shahrokni A, Korc-Grodzicki B (2015) Delirium in elderly patients undergoing intraabdominal cancer surgeryassociated factors and consequences. Eur Geriatr Med 6:S76

59. Waked WJ et al (2015) Recognizing encephalopathy and delirium in the cardiopulmonary rehabilitation setting. Rehabil Psychol 60(2):201-210

60. Inouye SK, Westendorp RG, Saczynski JS (2014) Delirium in elderly people. Lancet 383(9920):911-922

61. Hendry K et al (2016) Evaluation of delirium screening tools in geriatric medical inpatients: a diagnostic test accuracy study. Age Ageing 45(6):832-837

62. Hall RJ, Meagher DJ, MacLullich AM (2012) Delirium detection and monitoring outside the ICU. Best Pract Res Clin Anaesthesiol 26(3):367-383

63. De J, Wand AP (2015) Delirium screening: a systematic review of delirium screening tools in hospitalized patients. Gerontologist 55(6):1079-1099
64. Wong CL et al (2010) Does this patient have delirium? Value of bedside instruments. JAMA 304(7):779-786

65. Breitbart W et al (1997) The Memorial Delirium Assessment scale. J Pain Symptom Manag 13(3):128-137

66. Baird L, Spiller JA (2017) A quality improvement approach to cognitive assessment on hospice admission: could we use the 4AT or Short CAM? BMJ Open Qual 6(2):153

67. De J et al (2017) Validating the 4A's test in screening for delirium in a culturally diverse geriatric inpatient population. Int J Geriatr Psychiatry $32(12): 1322-1329$

68. Tieges $\mathrm{Z}$ et al (2021) Diagnostic accuracy of the 4AT for delirium detection in older adults: systematic review and meta-analysis. Age Ageing 50(3):733-743

69. Grassi L et al (2001) Assessing delirium in cancer patients: the Italian versions of the Delirium Rating Scale and the Memorial Delirium Assessment Scale. J Pain Symptom Manag 21(1):59-68

70. Mitchell AJ et al (2014) The Mini-Mental State Examination as a diagnostic and screening test for delirium: systematic review and meta-analysis. Gen Hosp Psychiatry 36(6):627-633

71. Sands MB et al (2021) "SQiD, the Single Question in Delirium; can a single question help clinicians to detect delirium in hospitalised cancer patients?" running heading Single Question in Delirium" (Bcan-D-20-01665). BMC Cancer 21(1):75

72. Sigurdardottir KR et al (2014) The European Association for Palliative Care basic dataset to describe a palliative care cancer population: results from an international Delphi process. Palliat Med 28(6):463-473

73. Tayjasanant S, Bruera E, Hui D (2016) How far along the disease trajectory? An examination of the time-related patient characteristics in the palliative oncology literature. Support Care Cancer 24(9):3997-4004

Publisher's Note Springer Nature remains neutral with regard to jurisdictional claims in published maps and institutional affiliations. 